\title{
Long-term changes after carotid stenting assessed by intravascular ultrasound and near-infrared spectroscopy
}

\author{
Cyril Štěchovský^, Petr Hájek, Robert Roland, Martin Horváth, Josef Veselka \\ Department of Cardiology, Second Faculty of Medicine, Charles University, University Hospital Motol, Prague, Czech Republic \\ Contributions: (I) Conception and design: C Štěchovský, P Hájek, J Veselka; (II) Administrative support: C Štěchovský, P Hájek, R Roland, M \\ Horváth; (III) Provision of study materials or patients: C Štěchovský, P Hájek, J Veselka; (IV) Collection and assembly of data: C Štěchovský, P \\ Hájek, M Horváth, J Veselka; (V) Data analysis and interpretation: C Štěchovský, P Hájek, J Veselka; (VI) Manuscript writing: All authors; (VII) Final \\ approval of manuscript: All authors. \\ Correspondence to: Cyril Štěchovský. Department of Cardiology, Motol University Hospital, V Uvalu 84, 150 06, Prague, Czech Republic. \\ Email: stechovsky@gmail.com.
}

\begin{abstract}
Background: Long-term effect of carotid stenting (CAS) on the stabilization of the plaque is almost unrecognized. Vascular healing and remodeling might seal the atherosclerotic plaque with neointimal hyperplasia decreasing the vulnerability. We aimed to assess long-term change in the lipid signal, stent and luminal dimensions and restenosis after CAS with the intravascular ultrasound (IVUS) and near-infrared spectroscopy (NIRS) imaging.

Methods: We performed follow-up angiography and NIRS-IVUS imaging of 58 carotid stents in 52 patients. Median time from CAS to the follow-up examination was 31 months (range, 5-56). The lipid signal of the stented segment was calculated from a NIRS-derived chemogram (a spectroscopic map) as the lipid core burden index (LCBI, a dimensionless number from 0 to 1,000). Planimetric and volumetric measurements from IVUS were performed to assess change in minimal stent area (MSA), minimal luminal area (MLA), stent and luminal volume, late stent expansion and percentage in-stent restenosis (ISR) volume. Results: During the follow-up period, the mean $( \pm$ SD) LCBI significantly decreased from $32 \pm 56$ to $17 \pm 27$ $(\mathrm{P}=0.002)$. The mean stent volume significantly increased from $717 \pm 302$ to $1,019 \pm 429 \mathrm{~mm}^{3}(\mathrm{P}<0.001)$ with mean stent expansion $43 \% \pm 24 \%$. The mean luminal volume increased from $717 \pm 302$ to $760 \pm 359 \mathrm{~mm}^{3}$ $(\mathrm{P}=0.025)$ due to ISR encroaching $26 \% \pm 15 \%$ of the stent volume.
\end{abstract}

Conclusions: Lipid signal decreased during the follow-up period suggesting stabilization of the plaque. Late stent expansion was balanced with neointimal hyperplasia.

Trial registration: The trial is registered under clinicaltrials.gov NCT03141580.

Keywords: Carotid stenting; intravascular ultrasound; near-infrared spectroscopy; lipid core burden index; plaque stabilization

Submitted Mar 17, 2021. Accepted for publication Sep 29, 2021.

doi: $10.21037 / \mathrm{cdt}-21-160$

View this article at: https://dx.doi.org/10.21037/cdt-21-160

\section{Introduction}

Carotid endarterectomy (CEA) and carotid artery stenting (CAS) are established treatments of symptomatic and asymptomatic carotid artery stenosis. Both procedures have specific risks and require different selection of patients and lesions (1). Despite this, randomized trials reported similar favorable long-term outcomes of both endarterectomy and

\footnotetext{
$\wedge$ ORCID: 0000-0002-5869-8590.
} 
stenting of carotid stenosis $(2,3)$.

We hypothesized that the protective mechanism of CAS might be attributable to long-term stabilization of the plaque sealed under the stent. Serial intravascular ultrasound (IVUS) observation of carotid self-expandable stents demonstrated late stent expansion and significant neointimal hyperplasia after 6 months (4). Catheter-based intravascular near-infrared spectroscopy (NIRS) is an imaging method that enables detection of a lipid signal from the atherosclerotic plaque $(5,6)$. NIRS combined with IVUS has been utilized to assess coronary atherosclerosis and the effect of various interventions on the lipid signal in coronary plaques (7-9). NIRS proved to be accurate in detecting lipid rich atheroma in endarterectomy specimens of human internal carotid artery compared to histology gold standard (10). NIRS-IVUS imaging of carotid arteries in vivo proved to be safe and capable of detection of lipid rich plaques and their distribution in carotid stenosis (11) and characterization of the composition of carotid stenosis and restenosis (12). NIRS-IVUS during CAS demonstrated effect of stenting on the lipid component of the plaque (13). CAS of the lipid rich plaque is associated with high risk of cerebral microembolization measured on postprocedural magnetic resonance imaging (14).

We conducted a prospective follow-up observational study with NIRS-IVUS imaging of implanted carotid stents on patients who underwent CAS at our institution and who had baseline intraprocedural NIRS-IVUS imaging. IVUS was used to assess late stent expansion and restenosis and NIRS to measure change in the lipid signal which is a surrogate of plaque vulnerability.

We present the following article in accordance with the STROBE reporting checklist (available at https://dx.doi. org/10.21037/cdt-21-160).

\section{Methods}

\section{Study design}

In a tertiary cardiovascular center, we prospectively enrolled patients who underwent CAS with implantation of selfexpandable stent for atherosclerotic internal carotid artery (ICA) stenosis from May 2013 to June 2018 and had baseline NIRS-IVUS imaging performed during the CAS procedure. Results of the periprocedural NIRS-IVUS imaging were published previously (13). The study was conducted in accordance with the Declaration of Helsinki (as revised in 2013), and was approved by institutional ethics committee of Motol University Hospital (Fakultní nemocnice v Motole) (No.: EK-953/16), and informed consent was taken from all individual participants. The study was registered with clinicaltrials.gov (identifier: NCT03141580).

The primary outcome measure was the change in the lipid signal of the stented segment of common and internal carotid artery (ICA) from baseline (measurement immediately after CAS) to the follow-up imaging. Lipid signal was measured as a lipid core burden index (LCBI) extracted from NIRS chemogram. The secondary measures were change in minimal stent area (MSA) and minimal luminal area (MLA), expansion of the stent measured as change of stent volume and restenosis.

\section{Study population and procedures}

Fifty-two patients with 58 implanted carotid stents who previously underwent CAS with periprocedural NIRSIVUS imaging at our institution were enrolled to the study. Median time from CAS to the follow-up examination was 31 months (range, 5-56 months). Basic demographic characteristics, medical history and laboratory tests for cholesterol, C-reactive protein and creatinine levels are summarized in Table 1 . None of the patients suffered from ipsilateral stroke during the follow-up period. The follow-up examination was performed during a 2-day hospitalization. Patients were not financially rewarded for the study participation. The follow-up procedure consisted of carotid angiography and NIRS-IVUS imaging of the implanted stent. Procedures were performed using a 6-French guiding catheter or a 6-French sheath introduced from the femoral artery. The antithrombotic regimen included administration of a bolus of heparin $(70 \mathrm{IU} / \mathrm{kg})$. NIRS-IVUS monorail catheter was advanced over a $0.014-$ inch hydrophilic wire without using a protection device (filter). There were no neurologic events at the time of follow-up angiography and NIRS-IVUS. All 58 NIRSIVUS imaging were successfully performed and were included into the final analysis.

\section{NIRS-IVUS imaging and analysis}

NIRS and IVUS were performed utilizing a single $3.2 \mathrm{~F}$ monorail catheter TVC-8 Insight Imaging System (Infraredx, Burlington, MA, USA), which enables simultaneous coregistration of both modalities (6). The system utilizes absorbance and backscattering of the near-infrared 
Table 1 Patient, lesion and procedural characteristics

\begin{tabular}{|c|c|}
\hline Characteristics & Value \\
\hline Patients & 52 \\
\hline Stents & 58 \\
\hline Median follow-up (months) & $35[5-51]$ \\
\hline Male & $35(67 \%)$ \\
\hline Age (years) & $67.4 \pm 8.1$ \\
\hline Ipsilateral stroke during follow-up & 0 \\
\hline Coronary artery disease & $32(62 \%)$ \\
\hline Diabetes & $19(37 \%)$ \\
\hline Arterial hypertension & $50(96 \%)$ \\
\hline Smoking & $26(50 \%)$ \\
\hline High dose statin* & $49(94 \%)$ \\
\hline ASA or clopidogrel & $47(90 \%)$ \\
\hline Anticoagulation & $8(15 \%)$ \\
\hline Total cholesterol (mmol/L) & $4.32 \pm 0.93$ \\
\hline LDL cholesterol (mmol/L) & $2.44 \pm 0.83$ \\
\hline Creatinine $(\mu \mathrm{mol} / \mathrm{L})$ & $84.8 \pm 24.8$ \\
\hline C-reactive protein (mg/L) & $4.8 \pm 8.9$ \\
\hline Open cell stents (Sinus) & $15(26 \%)$ \\
\hline Hybrid stents (Cristallo) & $3(5 \%)$ \\
\hline Closed cell stents (Xact, Wallstent) & $40(69 \%)$ \\
\hline Stent length (mm) & $37 \pm 5$ \\
\hline Distal nominal diameter of implanted stents $(\mathrm{mm})$ & $7.3 \pm 0.9$ \\
\hline
\end{tabular}

*, atorvastatin $40-80 \mathrm{mg}$ or rosuvastatin $20-40 \mathrm{mg}$ daily

wavelength to determine chemical composition (6). The result of NIRS examination is presented as a chemogram-a two-dimensional map with the $\mathrm{x}$-axis indicating the position of the measurement on the long axis of the vessel and the $y$-axis circumferential position of the measurement on the cross-section through the vessel-where every picture element (pixel) represents one spectroscopic measurement (Figure 1). Every measurement is binary coded as yellow (positive) or red (negative). The LCBI is a summary measure of the lipid signal along the interrogated segment of the vessel on a scale of 0 to 1,000 . The LCBI is calculated as a fraction of yellow pixels in the chemogram multiplied by a factor of 1,000 (6). The NIRS probe at the distal tip of the catheter acquires 40 spectroscopic measurements per second during automated pullback through the catheter at a speed of $0.5 \mathrm{~mm} / \mathrm{s}$ and 16 rotations per second. IVUS images are acquired via a $40 \mathrm{MHz}$ transducer rotating at the same speed as the NIRS probe.

Quantitative grayscale IVUS analysis was performed using the QIvus software (Medis, Leiden, the Netherlands). IVUS was used to assess distal reference diameter and area, MLA, MSA, stent and luminal volumes, in-stent restenosis (ISR) volume, residual stenosis area compared to the distal reference segment, late luminal area and volume gain and stent expansion. Measurements of the external elastic membrane (EEM) to assess plaque volume were not performed because acoustic shadowing of the stent and calcifications obscured the EEM border. All IVUS morphologic analysis, definitions and measurements follow expert consensus document of the American College of Cardiology (15). IVUS calculations are summarized in Table 2. Quantitative analysis of NIRS-derived chemograms was performed using the QIvus software (Medis, Leiden, the Netherlands). Chemograms were compared to assess the change in the lipid signal from the segment of ICA and CCA with implanted stent (i.e., region of interest; ROI) from baseline to the follow-up. The following parameters were used to quantify the lipid signal: LCBI of the entire stented segment and maximal LCBI in any 4-mm segment of the chemogram (LCBImax). LCBI and LCBImax were automatically calculated from the ROI defined by the distinct appearance of the stent on the coregistered IVUS image.

\section{Statistical analysis}

Statistical analyses were performed using the GraphPad Prism 8 software (GraphPad Software, La Jolla, CA, USA). Normally distributed continuous variables are presented as mean \pm standard deviation (SD) and compared with a Wilcoxon matched-pair signed rank test for paired samples. Categorical variables are presented as frequency (\%) and compared with Pearson's $\chi^{2}$ test. A linear association between continuous variables is expressed with correlation plots and Pearson's correlation coefficient (r). A two-sided $P$ value of 0.05 or less was considered to indicate statistical significance.

\section{Results}

\section{Stent and luminal measurements}

During the follow-up period, changes in the NIRS-IVUS measurements are summarized in Table 3. The mean MSA 


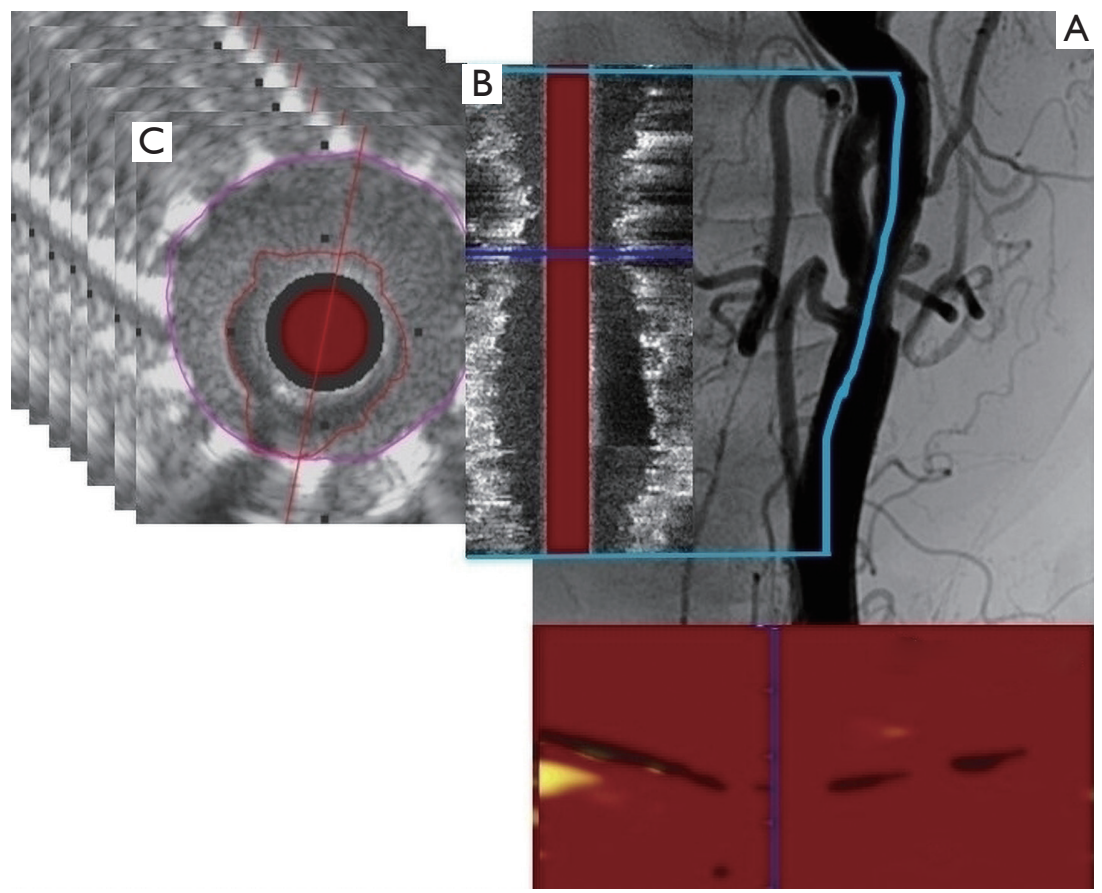

Figure 1 Angiogram of carotid artery with implanted stent (A) and corresponding longitudinal (B) and cross-sectional (C) IVUS imaging with NIRS-derived chemogram.

Table 2 IVUS definitions and calculations

\begin{tabular}{ll}
\hline IVUS variable & Calculation \\
\hline Residual stenosis area $(\%)$ & {$[1-($ MLA/distal reference area) $] \times 100$} \\
Stent expansion $(\%)$ & $\{[$ stent volume (follow-up)/stent volume (baseline) $]-1\} \times 100$ \\
In-stent restenosis volume $\left(\mathrm{mm}^{3}\right)$ & Stent volume (follow-up) - luminal volume (follow-up) \\
Percentage in-stent restenosis volume $(\%)$ & {$[$ In-stent restenosis volume/stent volume (follow-up) $\times \times 100$} \\
Late lumen gain/loss area $\left(\mathrm{mm}^{2}\right)$ & MLA (follow-up) - MLA (baseline) \\
Percentage late lumen gain/loss area $(\%)$ & $\{[$ MLA (follow-up)/MLA (baseline) $]-1\} \times 100$ \\
\hline
\end{tabular}

MLA, minimal luminal area.

significantly increased $(\mathrm{P}<0.001)$ which was compensated with restenosis. Therefore, there was no significant change in the MLA ( $\mathrm{P}=0.098)$ (Figure 2A,2B). The net MLA gain was $4 \pm 33 \%$ from the baseline value. There was no significant change of residual stenosis area $(\mathrm{P}=0.221)$. Using volumetric measurements, there was a significant increase in the luminal volume $(\mathrm{P}=0.025)$ (Figure $2 C)$. Stents expanded by $43 \% \pm 24 \%$ of their baseline volume (Figure $2 D$ ) with ISR encroaching $26 \% \pm 15 \%$ of the stent volume. The resulting net luminal volume gain was $6 \% \pm 30 \%$. There was no correlation between volumetric measurement of stent expansion and ISR (Pearson's correlation coefficient $\mathrm{r}=0.19 ; \mathrm{P}=0.16$ ) (Figure $3 A$ ) and a weak correlation between baseline LCBI and ISR (Pearson's correlation coefficient $\mathrm{r}=0.30 ; \mathrm{P}=0.024$ ) (Figure $3 B$ ). Time from CAS did not correlate with percentage ISR volume (Pearson's correlation coefficient $\mathrm{r}=-0.02 ; \mathrm{P}=0.88$ ) (Figure $4 A$ ) or stent expansion (Pearson's correlation coefficient $\mathrm{r}=0.11 ; \mathrm{P}=0.45$ ) (Figure $4 B$ ). No significant difference in the percentage ISR volume was observed between subgroups including stent 
Table 3 Change in the laboratory values and NIRS-IVUS measurements during the follow-up period

\begin{tabular}{|c|c|c|c|}
\hline Variable & Baseline & Follow-up & $P$ value \\
\hline Minimal stent area $\left(\mathrm{mm}^{2}\right)$ & $11.1 \pm 3.0$ & $15.0 \pm 3.5$ & $<0.001$ \\
\hline Luminal volume $\left(\mathrm{mm}^{3}\right)$ & $717 \pm 302$ & $760 \pm 359$ & 0.025 \\
\hline Stent volume $\left(\mathrm{mm}^{3}\right)$ & $717 \pm 302$ & $1,019 \pm 429$ & $<0.001$ \\
\hline Maximal lipid core burden index per $4 \mathrm{~mm}$ & $122 \pm 136$ & $80 \pm 111$ & 0.002 \\
\hline Total cholesterol (mmol/L) & $4.12 \pm 0.99$ & $4.11 \pm 0.89$ & 0.891 \\
\hline LDL-cholesterol (mmol/L) & $2.31 \pm 0.83$ & $2.27 \pm 0.73$ & 0.742 \\
\hline C-reactive protein (mg/L) & $5.1 \pm 9.2$ & $4.6 \pm 8.8$ & 0.455 \\
\hline
\end{tabular}

A

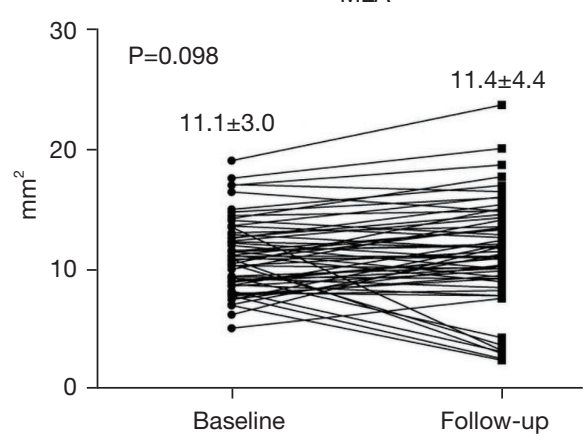

C

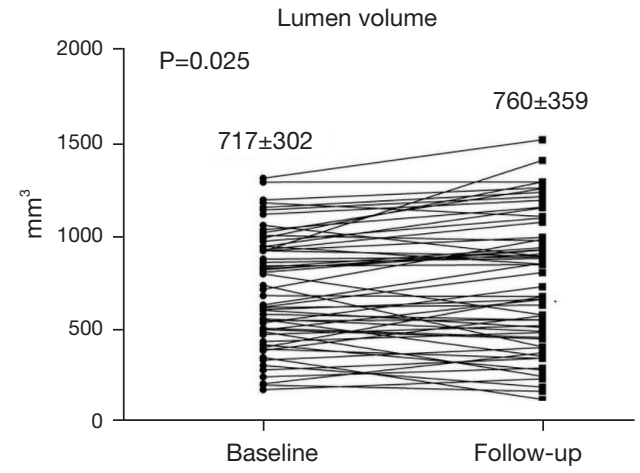

B

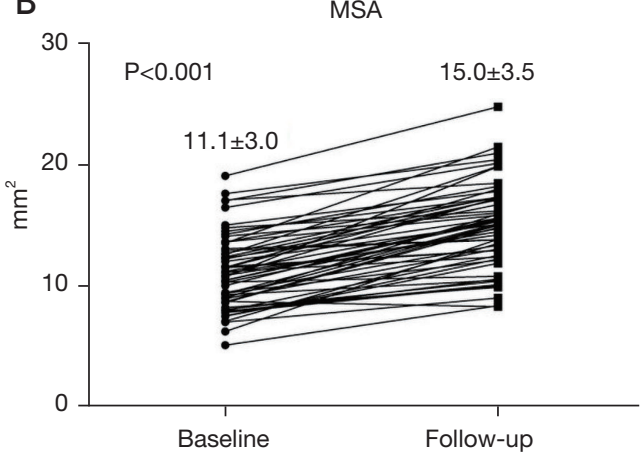

D

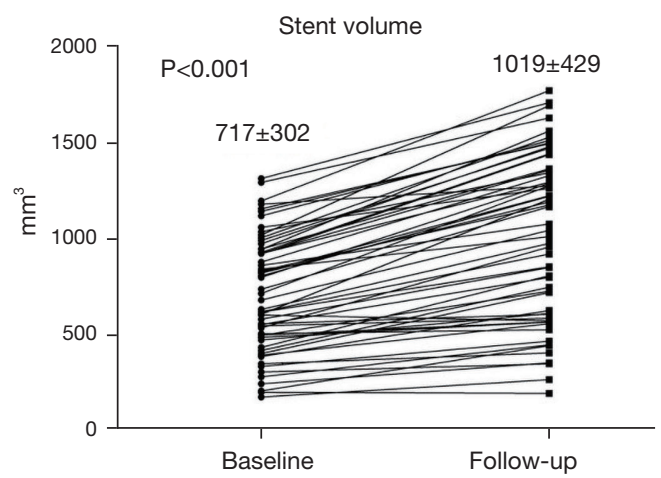

Figure 2 Quantitative IVUS assessment (mean \pm SD) of the change in (A) minimal luminal area, (B) minimal stent area, (C) luminal volume and (D) stent volume during the follow-up period. 

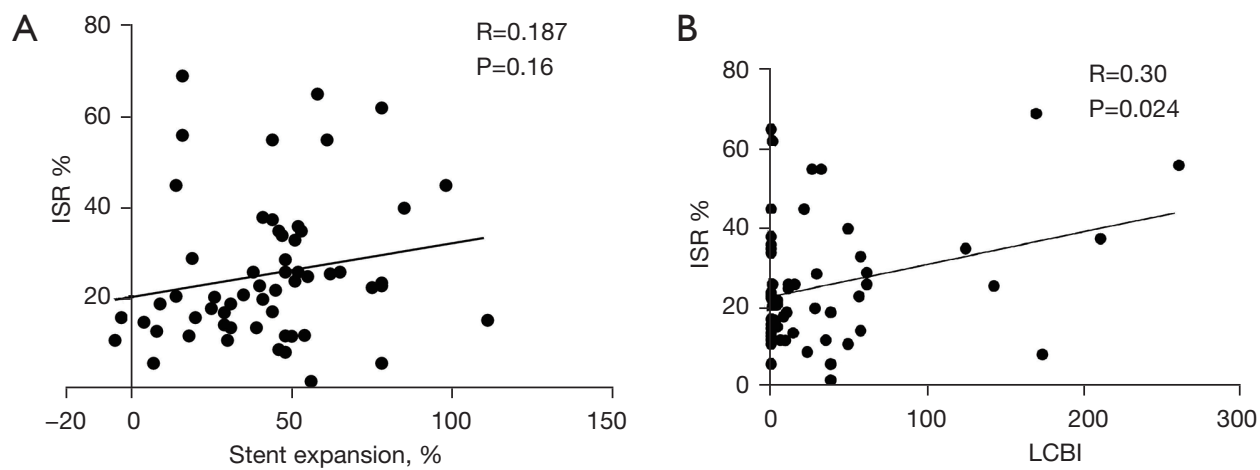

Figure 3 Scatter plots with Pearson's correlation between (A) percentage stent expansion and percentage in-stent restenosis (ISR) volume and (B) baseline lipid core burden index (LCBI) and percentage ISR volume.
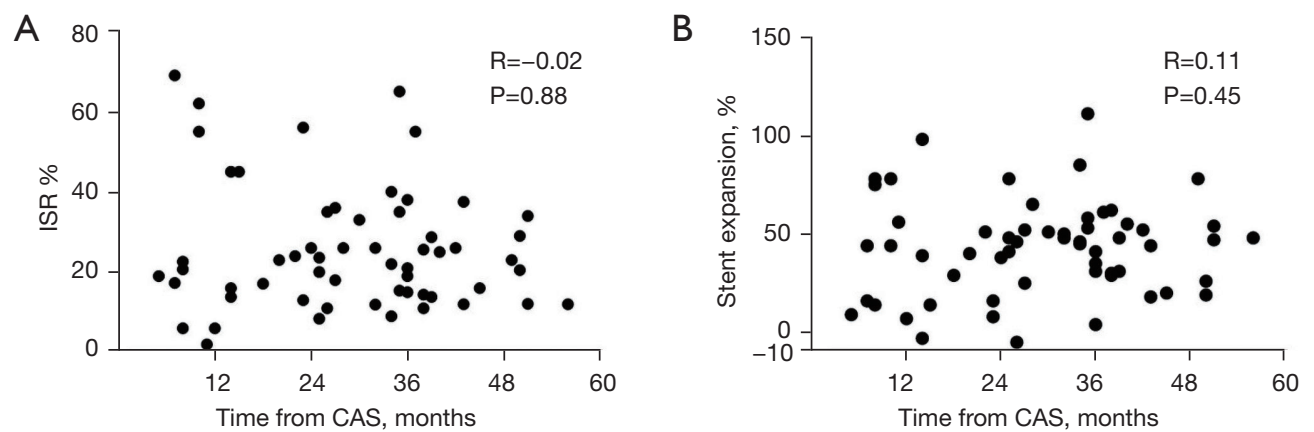

Figure 4 Scatter plots with Pearson's correlation between length of the follow-up and (A) percentage in-stent restenosis (ISR) volume and (B) stent expansion.

design (open versus closed cell) (Table 4).

\section{Lipid signal}

A significant decrease of LCBI $(\mathrm{P}=0.002)$ and maximal lipid core burden index per $4 \mathrm{~mm}(\mathrm{P}=0.002)$ was observed during follow-up (Figure 5A,5B). The follow-up LCBImax $<100$ was present in all but one case with no correlation between follow-up LCBI and ISR volume (Pearson's correlation coefficient $r=0.16 ; \mathrm{P}=0.25)$. There was no correlation between follow-up LCBI and luminal volume $(\mathrm{P}=0.72)$ or stent volume $(\mathrm{P}=0.34)$. Diabetics had higher follow-up LCBI compared to patients without diabetes $(\mathrm{P}=0.02)$ (Table 4). No significant difference in the follow-up LCBI was detected between other subgroups (Table 4).

\section{Clinical and procedural predictors of restenosis}

Significant luminal restenosis (reduction of MLA $\geq 50 \%$ from baseline) was present in 7 (12\%) stents. Stents with restenosis had smaller MLA at baseline $(9.15 \pm 2.27 \mathrm{vs}$. $\left.11.4 \pm 3.05 \mathrm{~mm}^{2}, \mathrm{P}=0.037\right)$ and generated significantly more percentage ISR volume $(53 \% \pm 17 \%$ vs. $22 \% \pm 11 \%, \mathrm{P}<0.001)$. No significant difference in percentage ISR volume was detected between sexes, diabetics, current smokers, patients on high dose statin therapy or stent designs (open versus closed cell) (Table 4).

\section{Discussion}

The present study used serial NIRS-IVUS imaging of carotid self-expanding stents to provide an insight on longterm remodeling and atherosclerotic plaque stabilization after CAS. To our knowledge it is the first study utilizing multimodality NIRS-IVUS imaging to assess long-term changes after CAS. The major findings are as follows: (I) significant reduction of lipid signal both though the entire stented segment and at the site with maximal LCBI (i.e., 
Table 4 Percentage in-stent restenosis volume and follow-up LCBI in different subgroups of patients

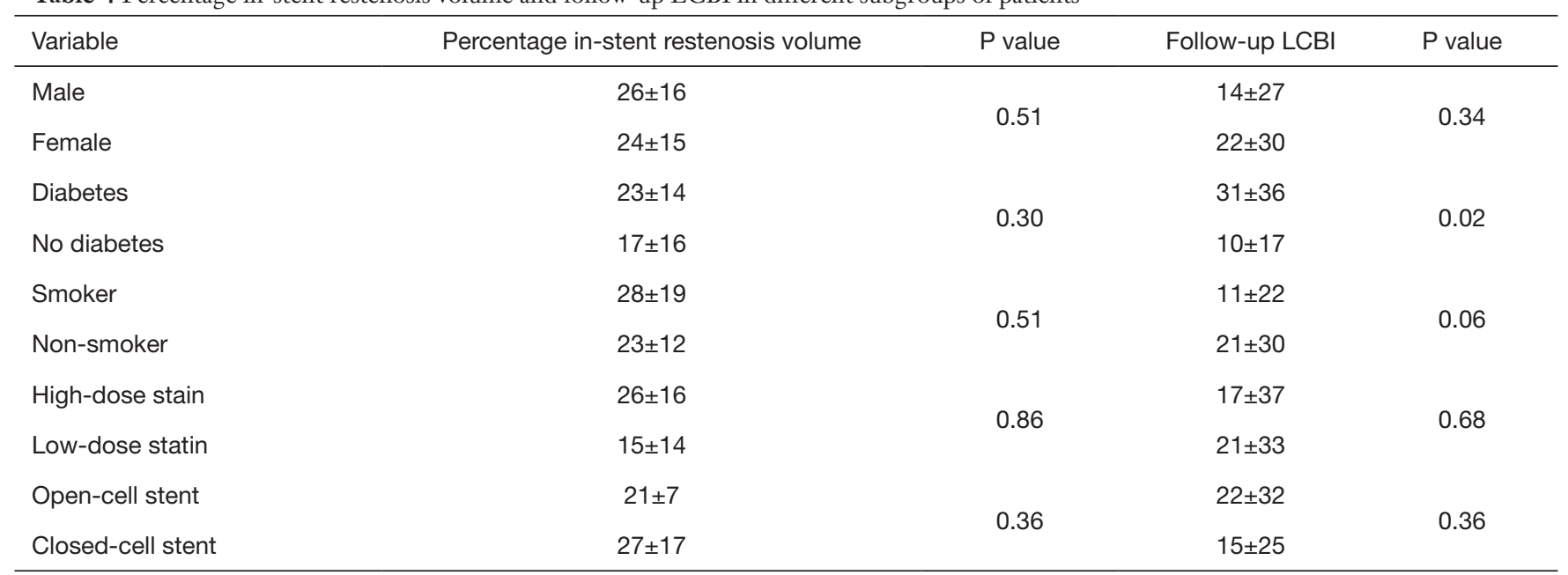

LCBI, lipid core burden index.

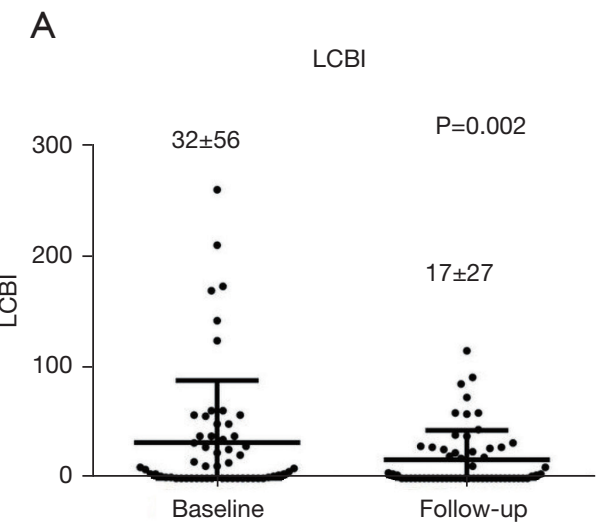

B

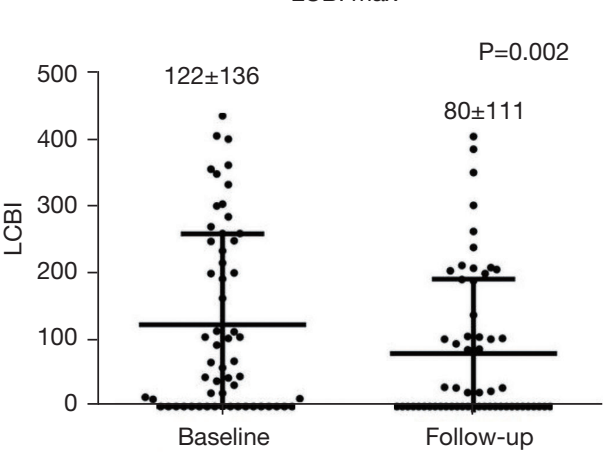

Figure 5 Quantitative NIRS assessment (mean \pm SD) of the change in lipid core burden index (LCBI) during the follow-up period (A) in the entire stented segment and (B) maximal LCBI in any 4-mm segment of the chemogram. The middle bar represents mean and the error bars represent standard deviation.

lipid core); (II) late stent expansion through the entire stented segment including the site with MLA; (III) marked neointimal hyperplasia attenuating the effect of late stent expansion on late luminal volume gain and completely neutralizing the effect on late MLA gain; (IV) significant restenosis is likely caused by small postprocedural MLA combined with excessive neointimal proliferation.

Although CAS has been introduced more than three decades ago the protective mechanism of CAS on the risk of ipsilateral stroke is not properly understood. Unlike other vascular territories where angioplasty and stenting relieve hemodynamic obstruction and thus improve symptoms carotid stenosis does not usually cause significant hemodynamic impact on cerebral circulation (16). Stroke attributable to carotid stenosis is caused by atherothrombosis and embolization from the plaque rather than hemodynamic deterioration. Whereas endarterectomy completely removes the atherosclerotic plaque which prevents embolization, stenting merely restores the luminal area but the plaque remains sealed under the stent. Previous studies suggested that there is ongoing healing process starting the first day after CAS with submillimeter thin echolucent layer between the stent and the luminal perfused area which might be interpreted as a mural thrombus (17). In follow-up this layer increases in thickness (up to 6 months) and echogenicity (up to 12 months) which was interpreted as neointimal 
ingrowth (18). This healing process leads to neointimal coverage of the stent and the atherosclerotic plaque $(4,17,18)$. Added value of our study is that we not only assessed long-term stent expansion and restenosis through the entire stented segment with volumetric calculations but also composition of the restenotic tissue by comparing the postprocedural and follow-up lipid signal which is a marker of vulnerability of the lesion.

Reduction of the lipid signal might be caused by the change of the composition of the atherosclerotic plaque under the stent, the effect of high dose statin therapy. Reduction of LCBI in coronary arteries has been reported after intensive statin therapy (19). Stable or reduced LCBI on the follow-up imaging in most cases suggests that neointimal hyperplasia (NIH) is responsible for most of the ISR. Reduction of lipid signal might also represent NIH sealing the atherosclerotic plaque from the luminal surface. Chemogram does not allow to determine the depth from which the wavelength spectra indicating cholesterol presence are reflected $(5,6)$. Therefore, chemogram does not directly detect thickness of the fibrous cap covering the lipidic core $(5,6)$. Nonetheless, it has been repeatedly demonstrated that high LCBI (i.e., lipid rich plaque) represents a vulnerable plaque and low LCBI a stable lesion (7-10,20-23). High LCBI also correlates with thin cap fibroatheroma detected on optical coherence tomography imaging (24). Nakagawa et al. demonstrated that high LCBI represents a vulnerable lesion for CAS (14). Patients with lipid rich plaque in the ICA had high risk of asymptomatic cerebral embolization assessed by postprocedural magnetic resonance (MR) imaging. In their study NIRS was significantly better predictor of microembolization than IVUS or MR of the carotid plaque. In our observation NIH covered the stent which was accompanied by decreased lipid signal, although the causality could not be determined due to the reasons mentioned former. The mechanism of plaque sealing might be responsible for low incidence of ipsilateral stroke after 30-days post successful CAS comparable to complete removal of the plaque with endarterectomy $(2,3)$.

Our finding of $43 \%$ late stent expansion and 26\% ISR volume corresponds closely to a previously published study of serial IVUS imaging of carotid stents by Clark where the late MSA enlargement was $49 \%$ and ISR area at the same site was $37 \%$ (4). The difference was in median time from CAS which was 31 months in our study and 6 months in the study by Clark and in the methods of measurements. We used volumetric calculations which reflect changes in the entire stented segment of ICA. Similarly with Clark, we did not find correlation between percentage stent expansion and percentage ISR (4). Serial IVUS studies on coronary arteries revealed that self-expanding coronary stents continue to expand but generate significantly more ISR than balloon-expanding stents $(25,26)$. The likely explanation for late stent expansion with marked neointimal proliferation is that the stents are oversized (mean stent diameter was $7.3 \pm 0.9 \mathrm{~mm}$ compared to diameter of distal ICA $4.3 \pm 0.8 \mathrm{~mm}$ in the study) which causes ongoing chronic injury promoting NIH (27). Other factors probably contribute to the amount of stent expansion (e.g., calcification, stent design with different radial force) and neointimal proliferation (e.g., inflammatory milieu) which results in large interindividual variability of stent expansion and ISR. However, limited size of the study did not allow to assess for clinical and procedural characteristics causing variable response to CAS.

\section{Limitations}

This is an observational study with limited sample size. Significant clinical and procedural predictors of ISR and LCBI change might have been detected with a much larger number of patients. Only one NIRS-IVUS followup imaging was performed at different time intervals from CAS (median 31 months). Longer follow-up was not correlated with higher percentage ISR volume or larger stent expansion. Therefore, the temporal trend of this processes could not be clarified. Previous study suggested that most neointimal hyperplasia occurs in the first 12 months after CAS $(17,18)$. It is not feasible to perform multiple invasive carotid imaging procedures in a single patient. Duplex ultrasound should be used for this purpose $(17,18)$. Detection of lipid core with NIRS was not compared with another imaging method because there is no gold standard for plaque composition in vivo. However, the accuracy of NIRS has been recently validated on human carotid artery plaques (10). Computed tomography and magnetic resonance imaging are used to characterize carotid plaques, but their performance is largely limited by the implanted stent. Both ultrasound and near-infrared light can be obstructed by stent, although with IVUS we assessed processes within the stent and with NIRS signal obstruction in present both at baseline and follow-up.

\section{Conclusions}

Although self-expanding carotid stents continued to expand 
after CAS, they also generated considerable amount of neointimal hyperplasia. Therefore, restenosis was balanced with continuous stent expansion. Lipid signal from the stented segment significantly decreased during the longterm follow-up suggesting stabilization of the plaque.

\section{Acknowledgments}

Funding: This work was supported by the Project for Conceptual Development of Research Organization No. 00064203 from Ministry of Health, Czech Republic.

\section{Footnote}

Reporting Checklist: The authors have completed the STROBE reporting checklist. Available at https://dx.doi. org/10.21037/cdt-21-160

Data Sharing Statement: Available at https://dx.doi. org/10.21037/cdt-21-160

Peer Review File: Available at https://dx.doi.org/10.21037/ cdt-21-160

Conflicts of Interest: All authors have completed the ICMJE uniform disclosure form (available at https://dx.doi. org/10.21037/cdt-21-160). The authors have no conflicts of interest to declare.

Ethical Statement: The authors are accountable for all aspects of the work in ensuring that questions related to the accuracy or integrity of any part of the work are appropriately investigated and resolved. The study was conducted in accordance with the Declaration of Helsinki (as revised in 2013). The study was approved by institutional ethics committee of Motol University Hospital (Fakultní nemocnice v Motole) (No.: EK-953/16) and informed consent was taken from all individual participants.

Open Access Statement: This is an Open Access article distributed in accordance with the Creative Commons Attribution-NonCommercial-NoDerivs 4.0 International License (CC BY-NC-ND 4.0), which permits the noncommercial replication and distribution of the article with the strict proviso that no changes or edits are made and the original work is properly cited (including links to both the formal publication through the relevant DOI and the license). See: https://creativecommons.org/licenses/by-nc-nd/4.0/.

\section{References}

1. White CJ. Carotid artery stenting. J Am Coll Cardiol 2014;64:722-31.

2. Rosenfield K, Matsumura JS, Chaturvedi S, et al. Randomized Trial of Stent versus Surgery for Asymptomatic Carotid Stenosis. N Engl J Med 2016;374:1011-20.

3. Brott TG, Howard G, Roubin GS, et al. Long-Term Results of Stenting versus Endarterectomy for CarotidArtery Stenosis. N Engl J Med 2016;374:1021-31.

4. Clark DJ, Lessio S, O'Donoghue M, et al. Mechanisms and predictors of carotid artery stent restenosis: a serial intravascular ultrasound study. J Am Coll Cardiol 2006;47:2390-6.

5. Gardner CM, Tan H, Hull EL, et al. Detection of lipid core coronary plaques in autopsy specimens with a novel catheter-based near-infrared spectroscopy system. JACC Cardiovasc Imaging 2008;1:638-48.

6. Waxman S, Dixon SR, L'Allier P, et al. In vivo validation of a catheter-based near-infrared spectroscopy system for detection of lipid core coronary plaques: initial results of the SPECTACL study. JACC Cardiovasc Imaging 2009;2:858-68.

7. Stone GW, Maehara A, Muller JE, et al. Plaque Characterization to Inform the Prediction and Prevention of Periprocedural Myocardial Infarction During Percutaneous Coronary Intervention: The CANARY Trial (Coronary Assessment by Near-infrared of Atherosclerotic Rupture-prone Yellow). JACC Cardiovasc Interv 2015;8:927-36.

8. Madder RD, Goldstein JA, Madden SP, et al. Detection by near-infrared spectroscopy of large lipid core plaques at culprit sites in patients with acute ST-segment elevation myocardial infarction. JACC Cardiovasc Interv 2013;6:838-46.

9. Oemrawsingh RM, Cheng JM, García-García HM, et al. Near-infrared spectroscopy predicts cardiovascular outcome in patients with coronary artery disease. J Am Coll Cardiol 2014;64:2510-8.

10. Kotsugi M, Nakagawa I, Hatakeyama K, et al. Lipid Core Plaque Distribution Using Near-infrared Spectroscopy Is Consistent with Pathological Evaluation in Carotid Artery Plaques. Neurol Med Chir (Tokyo) 2020;60:499-506.

11. Štěchovský C, Hájek P, Horváth M, et al. Near-infrared spectroscopy combined with intravascular ultrasound in carotid arteries. Int J Cardiovasc Imaging 2016;32:181-8.

12. Štěchovský C, Hájek P, Horváth $M$, et al. Composition of 
carotid artery stenosis and restenosis: A series of patients assessed with intravascular ultrasound and near-infrared spectroscopy. Int J Cardiol 2016;207:64-6.

13. Štěchovský C, Hájek P, Horváth M, et al. Effect of stenting on the near-infrared spectroscopy-derived lipid core burden index of carotid artery plaque. EuroIntervention 2019;15:e289-96.

14. Nakagawa I, Kotsugi M, Park HS, et al. Near-infrared spectroscopy carotid plaque characteristics and cerebral embolism in carotid artery stenting. EuroIntervention 2021;17:599-606.

15. Mintz GS, Nissen SE, Anderson WD, et al. American College of Cardiology Clinical Expert Consensus Document on Standards for Acquisition, Measurement and Reporting of Intravascular Ultrasound Studies (IVUS). A report of the American College of Cardiology Task Force on Clinical Expert Consensus Documents. J Am Coll Cardiol 2001;37:1478-92.

16. Shakur SF, Hrbac T, Alaraj A, et al. Effects of extracranial carotid stenosis on intracranial blood flow. Stroke 2014;45:3427-9.

17. Willfort-Ehringer A, Ahmadi R, Gschwandtner ME, et al. Healing of carotid stents: a prospective duplex ultrasound study. J Endovasc Ther 2003;10:636-42.

18. Willfort-Ehringer A, Ahmadi R, Gruber D, et al. Arterial remodeling and hemodynamics in carotid stents: a prospective duplex ultrasound study over 2 years. J Vasc Surg 2004;39:728-34.

19. Kini AS, Baber U, Kovacic JC, et al. Changes in plaque lipid content after short-term intensive versus standard statin therapy: the YELLOW trial (reduction in yellow plaque by aggressive lipid-lowering therapy). J Am Coll Cardiol 2013;62:21-9.

20. Waksman R, Di Mario C, Torguson R, et al. Identification of patients and plaques vulnerable to future coronary events with near-infrared spectroscopy intravascular ultrasound imaging: a prospective, cohort study. Lancet 2019;394:1629-37.

21. Madder RD, Husaini M, Davis AT, et al. Large lipid-rich coronary plaques detected by near-infrared spectroscopy at non-stented sites in the target artery identify patients likely to experience future major adverse cardiovascular events. Eur Heart J Cardiovasc Imaging 2016;17:393-9.

22. Schuurman AS, Vroegindewey M, Kardys I, et al. Nearinfrared spectroscopy-derived lipid core burden index predicts adverse cardiovascular outcome in patients with coronary artery disease during long-term follow-up. Eur Heart J 2018;39:295-302.

23. Kini AS, Motoyama S, Vengrenyuk Y, et al. Multimodality Intravascular Imaging to Predict Periprocedural Myocardial Infarction During Percutaneous Coronary Intervention. JACC Cardiovasc Interv 2015;8:937-45.

24. Roleder T, Kovacic JC, Ali Z, et al. Combined NIRS and IVUS imaging detects vulnerable plaque using a single catheter system: a head-to-head comparison with OCT. EuroIntervention 2014;10:303-11.

25. Han RO, Schwartz RS, Kobayashi Y, et al. Comparison of self-expanding and balloon-expandable stents for the reduction of restenosis. Am J Cardiol 2001;88:253-9.

26. Kobayashi Y, Honda Y, Christie GL, et al. Long-term vessel response to a self-expanding coronary stent: a serial volumetric intravascular ultrasound analysis from the ASSURE Trial.A Stent vs. Stent Ultrasound Remodeling Evaluation. J Am Coll Cardiol 2001;37:1329-34.

27. Schwartz RS. Pathophysiology of restenosis: interaction of thrombosis, hyperplasia, and/or remodeling. Am J Cardiol 1998;81:14E-7E.
Cite this article as: Štěchovský C, Hájek P, Roland R, Horváth M, Veselka J. Long-term changes after carotid stenting assessed by intravascular ultrasound and near-infrared spectroscopy. Cardiovasc Diagn Ther 2021;11(6):1180-1189. doi: $10.21037 / \mathrm{cdt}-21-160$ 